Revista Bioética

\title{
PESQUISA
}

\section{Desafios em saúde mental durante a pandemia: relato de experiência}

Alfredo José Minervino ${ }^{1}$, Marina Barbosa de Oliveira ${ }^{1}$, Kaio Aranda Lima da Cunha ${ }^{1}$, Ygor Thalles Almeida Bereza ${ }^{1}$

1. Universidade Federal da Paraíba, João Pessoa/PB, Brasil.

\section{Resumo}

A readequação do atendimento em saúde mental durante a pandemia de covid-19 foi facilitada por mudanças na legislação médica vigente. O teleatendimento trouxe oportunidades para tratar, acompanhar e identificar os transtornos mentais de modo diferente. Este artigo tem por objetivo relatar a experiência do serviço de saúde mental de um hospital universitário e da residência médica em psiquiatria durante este período. A assistência ambulatorial incluiu teleatendimento e consultas presenciais conforme a gravidade, e foi criado o projeto Acolhimento em Saúde Mental em Tempos de Pandemia para assistir os profissionais do hospital. Espera-se que no futuro o teleatendimento seja usado como ferramenta para o cuidado em saúde mental, sendo ainda necessário treinar os profissionais e avaliar a qualidade desse recurso.

Palavras-chave: Pandemias. Infecções por coronavírus. Saúde mental. Consulta remota.

\section{Resumen}

\section{Desafíos en salud mental durante la pandemia: informe de experiencia}

La adaptación de la atención de la salud mental durante la pandemia de covid-19 se vio facilitada por cambios en la legislación médica actual. La telemedicina ha brindado oportunidades para tratar, controlar e identificar trastornos mentales de otro modo. Este artículo tiene como objetivo relatar la experiencia del servicio de salud mental de un hospital universitario y de la residencia médica en psiquiatría durante este período. La atención ambulatoria incluyó telemedicina y consultas presenciales según la gravedad, y se creó el proyecto "Acogiendo la Salud Mental en Tiempos de Pandemia" para ayudar a los profesionales del hospital. Se espera que en el futuro se utilice la telemedicina como herramienta para la atención de la salud mental. Para ello, será necesario formar profesionales y evaluar la calidad de este recurso.

Palabras clave: Pandemias. Infecciones por coronavirus. Salud mental. Consulta remota.

\section{Abstract \\ Mental health challenges during the pandemic: an experience report}

The readjustment in the care of mental health patients during the Covid-19 pandemic was facilitated by changes in the current medical legislation. Telemedicine brought forth opportunities to treat, monitor and identify mental disorders in different ways. This article reports the experience of the mental health service in a teaching hospital and psychiatry residency program during this period. Outpatient care included telemedicine appointments and face-to-face visits, according to severity criteria, and the project Embracing Mental Health in Times of Pandemic was created to assist hospital health staff. The prospect for the future is the use of teleservice as a tool for mental health care, which requires better training for professionals and evaluating the quality of this modality. Keywords: Pandemics. Coronavirus infections. Mental health. Remote consultation. 


\section{Saúde mental e pandemia de covid-19}

As medidas de distanciamento social são essenciais para reduzir a velocidade de propagação do novo coronavírus (covid-19). Para isso, além de reorganizar recursos financeiros e redistribuir leitos de internação hospitalar, insumos e recursos humanos, os serviços de saúde optaram por suspender consultas ambulatoriais eletivas ${ }^{1}$. A inviabilidade de extinguir esta modalidade de atendimento levou o Conselho Federal de Medicina (CFM) a reconhecer excepcionalmente a responsabilidade e eticidade do uso temporário da telemedicina, com orientação, monitoramento e interconsulta remotos, durante a pandemia ${ }^{2}$.

Modificações importantes na legislação brasileira viabilizaram ainda a prescrição eletrônica ${ }^{3}$, a entrega em domicílio de medicamentos sujeitos a controle especial e a dispensação de mais remédios de um único receituário ${ }^{4}$. Experiências prévias sustentam a eficácia do teleatendimento em saúde mental em casos de depressão ${ }^{5}$, ansiedade ${ }^{6}$ e transtorno de estresse pós-traumático (TEPT) ${ }^{7}$. $\mathrm{O}$ uso deste recurso aumentou em todo o mundo, e muitos países o adotaram priorizando as pessoas com maior risco de exposição à covid-19 ${ }^{8}$. Primeiros relatos mostram que sobretudo pessoas isoladas procuravam ativamente suporte on-line em saúde mental ${ }^{9}$.

Países com recursos financeiros escassos têm priorizado medidas de controle da transmissão e manejo das formas graves da doença. Entretanto, a pandemia pode impactar severamente a situação econômica, social e de promoção da saúde em longo prazo ${ }^{10}$. Doentes psiquiátricos e populações vulneráveis necessitam de atenção em saúde mental qualificada em virtude do risco de agravamento da doença de base e surgimento de comorbidades psiquiátricas, como o TEPT, quadros graves de ansiedade e depressão, transtornos do sono e suicídio ${ }^{11}$.

Fatores sociais, como abandono familiar e habitação precária, podem dificultar a adesão de pacientes com transtornos mentais graves (TMG) às medidas de isolamento social, tornando-os assim mais vulneráveis à contaminação. Além disto, doenças como esquizofrenia e transtorno afetivo bipolar são associadas a prejuízos cognitivos e menor escolaridade, que inviabilizam a adesão a medidas básicas de prevenção, como a higiene ${ }^{12}$. A dificuldade em acompanhar e interpretar informações obtidas na mídia pode ainda agravar quadros psiquiátricos pré-existentes e dificultar a adesão ao tratamento, especialmente entre os agudamente enfermos.
O uso de tabaco pode ser também fator de risco para o surgimento de formas graves de covid-19 nesta população. Estima-se que cerca de $64 \%$ dos esquizofrênicos e $44 \%$ dos acometidos por transtorno afetivo bipolar fumem ${ }^{13}$. 0 tabagismo aumenta o risco por elevar a exposição a fômites, e acredita-se ainda que fumantes apresentem maior expressão da enzima conversora da angiotensina tipo 2 no epitélio brônquico, que pode estar relacionada ao aumento do risco de desenvolver a doença ${ }^{14}$.

Somados aos fatores inerentes ao paciente, os serviços de saúde mental são também mais propícios à contaminação porque em sua maioria são projetados de forma a priorizar a interação social. Ademais, de maneira geral, é raro que se exija nestes ambientes o uso de equipamentos de proteção individual, que podem estar pouco disponíveis, e a equipe pode não ter habilidade para sua correta utilização ${ }^{12}$. Fatores de risco para o desenvolvimento de formas graves de infecção por covid-19, como doenças cardiovasculares, diabetes e doença pulmonar obstrutiva crônica, também são mais associados a TMG, cujos portadores já apresentam taxa de mortalidade 3,7 vezes maior que a população em geral ${ }^{15}$.

A dificuldade de acesso a serviços especializados em saúde mental de emergência durante o período de isolamento, o estigma vivido pelos que padecem de transtornos mentais e a falta de treinamento dos serviços de emergências clínicas para atender a esta população favorecem o surgimento de uma epidemia de transtornos mentais concomitante ou subsequente à da covid-19 ${ }^{16}$. Neste cenário, a Associação Brasileira de Psiquiatria $(A B P)^{17}$ recomendou que serviços de emergências psiquiátricas já existentes permanecessem abertos e que novos locais fossem criados para conduzir pacientes em surto ou crise. Ressaltou ainda a necessidade de treinar as equipes de saúde em emergências psiquiátricas, especialmente as do Serviço de Atendimento Móvel de Urgência (Samu), com suporte remoto do médico psiquiatra quanto à melhor forma de encaminhar o paciente para atendimento ${ }^{17}$.

Visando evitar a disseminação do coronavírus entre pacientes internados em leitos psiquiátricos, a $A B P^{17}$ recomendou ainda internação domiciliar quando possível, brevidade da internação hospitalar, uso contínuo de máscara em pacientes sintomáticos e redução da frequência de visitas de familiares. Para os Centros de Atenção Psicossocial, aconselhou suspender 
atividades em grupo e reduzir o número de consultas individuais para o mínimo necessário ao usuário. No que se refere ao atendimento em psiquiatria infantil, orientou a remoção de brinquedos, avaliação prioritária da escuta dos pais antes do atendimento à criança, intenso cuidado com higienização de superfícies e uso de brincadeiras avaliativas evitando contato próximo ${ }^{18}$.

Além de se adequar às recomendações do Ministério da Saúde, do CFM e da ABP, o serviço de psiquiatria do Hospital Universitário Lauro Wanderley (SP-HULW) aderiu de forma prioritária à modalidade de teleatendimento, reservando a assistência ambulatorial aos casos de descompensação dos TMG. Desenvolveu ainda plano de ações para orientar seus funcionários quanto a prevenção e tratamento precoce de transtornos mentais relacionados ao período pandêmico.

Com isso, visando descrever precisamente a vivência desse serviço de forma a contribuir com a área de atuação, este artigo relata a experiência do funcionamento do serviço de saúde mental de hospital universitário e residência médica em psiquiatria durante a pandemia de covid-19. Tais descrições não necessariamente precisam ser exitosas, desde que contribuam para melhorar a prática ou sejam relevantes para o cuidado em saúde ${ }^{19}$.

\section{Readequação do atendimento durante a pandemia}

Diversas dificuldades têm sido enfrentadas para manter os serviços de saúde mental, como o afastamento de funcionários que se enquadram no grupo de risco e o deslocamento de pessoal para áreas de atenção consideradas prioritárias. Com vistas a garantir a assistência, o SP-HULW criou frentes emergenciais de trabalho relacionadas a atenção ao paciente em internação psiquiátrica, interconsulta, acolhimento e serviço ambulatorial especial e busca ativa, prevendo ações durante e após o período de isolamento social.

Durante a primeira fase tem-se garantido visitas de especialistas aos leitos de responsabilidade da residência médica do hospital psiquiátrico estadual, interconsultas aos pacientes de outras especialidades internados no hospital universitário e fornecimento de receitas e atestados para pacientes acompanhados no serviço ambulatorial. Mantêm-se também a avaliação contínua da necessidade de atendimento presencial ou remoto de pacientes por meio de busca ativa em prontuários e contato telefônico, e o atendimento aos profissionais do hospital no âmbito do projeto Acolhimento em Saúde Mental em Tempos de Pandemia. Visando informar a população e melhorar o enfrentamento da situação, temas relevantes em saúde mental têm sido ainda divulgados nas redes sociais, promovendo-se também atividades teóricas e discussões de casos relevantes para capacitar a equipe por meio de aplicativos.

Após o período de isolamento, o serviço retomará os atendimentos presenciais obedecendo a critérios de gravidade para agendar consultas com a equipe multiprofissional. Serão feitos levantamentos epidemiológicos pertinentes após o período de pandemia, e em seguida as atividades regulares serão retomadas.

\section{Atendimento ao paciente internado: interconsultas e psicofármacos}

A atenção a portadores de TMG que necessitam de internações hospitalares tem sido grande desafio durante a pandemia em decorrência do alto risco de contaminação coletiva pela covid-19 nestes estabelecimentos. Além disso, esses pacientes têm dificuldades de seguir orientações para controle da disseminação em ambientes compartilhados e, em alguns casos, de executar medidas básicas de higiene, como lavar as mãos. Ademais, o reconhecimento dos sintomas clínicos pode ser postergado pela incapacidade do paciente de identificá-los e relatá-los à equipe de saúde ${ }^{12}$. Fatores inerentes ao tratamento psiquiátrico, como o uso de clozapina - antipsicótico atípico utilizado em casos de esquizofrenia refratária -, podem ainda resultar em imunossupressão e aumentar a susceptibilidade a infecções graves ${ }^{20}$.

Visando reduzir o risco de disseminação e contaminação por covid-19, o uso de equipamentos de proteção individual pela equipe médica passou a ser obrigatório, reduzindo-se ainda a escala de profissionais e adotando-se a teleinterconsulta quando necessário. Tem sido observado que a restrição do acesso aos familiares, apesar de limitar a circulação de pessoas, dificulta a obtenção de informações relevantes sobre o paciente, a elaboração de plano de cuidados e a programação da alta precoce.

No hospital psiquiátrico estadual no qual também atua a residência médica do SP-HULW, área isolada e reservada foi criada para acolher pacientes sintomáticos respiratórios durante a internação, e indivíduos acometidos pelas formas graves da 
doença eram encaminhados para hospitais gerais. Quando necessitavam de avaliação psiquiátrica, os pacientes de outras especialidades clínicas, internados no hospital universitário relatado neste estudo, com ou sem infecção confirmada por covid-19, foram assistidos via interconsultas presenciais programadas.

Enfermos e familiares de portadores da doença do coronavírus também foram incluídos no plano de assistência como população de risco para desenvolvimento de transtornos mentais, uma vez que sofrem com a incerteza relacionada ao prognóstico e com a inexistência de tratamentos específicos para a doença. Este grupo é ainda exposto a situações traumáticas durante a internação e tem maior risco de desenvolver TEPT e sintomas depressivos e ansiosos que a população em geral ${ }^{21}$.

A segurança do uso de psicofármacos em pessoas infectadas por covid-19 ainda não foi estabelecida. Ademais, apesar de o tropismo do vírus pelo sistema nervoso central ter sido evidenciado, suas consequências em longo prazo são desconhecidas ${ }^{22}$. Na assistência ao portador da doença, o uso desses medicamentos tem sido guiado principalmente pelo perfil de interação medicamentosa.

Quando necessários, são preferíveis os seguintes antidepressivos: agomelatina, bupropiona, duloxetina, fluoxetina, fluvoxamina, paroxetina e sertralina. Antidepressivos tricíclicos, citalopram, mirtazapina e venlafaxina devem ser evitados pelo risco de aumento do intervalo QT/PR observado no eletrocardiograma ${ }^{23}$. Dos antipsicóticos, aripiprazol e lurasidona apresentam menor risco de alterações eletrocardiográficas, enquanto quetiapina e pimozida não são recomendadas pelo maior risco de interações medicamentosas. O uso de lítio e valproato como estabilizadores do humor parece seguro, mas a carbamazepina deve ser evitada pelo grande potencial de interação com outras drogas. O benzodiazepínico de preferência é o lorazepam, quando indicado ${ }^{23}$.

\section{Atendimento ambulatorial especial}

Para facilitar o acesso do usuário ao SP-HULW durante a pandemia, foram disponibilizados e divulgados nas mídias sociais oficiais números de telefone para contato com diversas especialidades médicas. Consultas - presenciais ou remotas, conforme avaliação em triagem presencial ou busca ativa - foram garantidas aos enfermos que estavam em acompanhamento regular no hospital. A demanda dos pacientes ambulatoriais que procuravam o hospital presencialmente era acoIhida, e o paciente era triado pelo enfermeiro de acordo com a classificação de risco para condições médicas psiquiátricas, com base em adaptações do protocolo de Manchester ${ }^{24}$.

Caso o paciente interrompesse o uso da medicação, relatasse efeitos colaterais relacionados aos psicofármacos prescritos pelo médico assistente do serviço de psiquiatria, quando sua demanda não fosse contemplada no protocolo ou nos casos em que o enfermeiro observasse possíveis emergências psiquiátricas, os médicos presentes no ambulatório tinham autonomia para realizar atendimento presencial imediato. $\mathrm{Na}$ impossibilidade de sanar a demanda ou na ausência de médico no momento da solicitação, o responsável pela triagem encaminhava o paciente para o serviço municipal de pronto atendimento em saúde mental por meio do Samu.

Pacientes assistidos no serviço ambulatorial foram contatados por telefone em buscas ativas com intuito de avaliar sintomas, acolher e triar necessidades, em processo conhecido como telemonitoramento e teleorientação. Quando eram identificadas descompensações graves, mas que não caracterizavam emergências psiquiátricas, o paciente era encaminhado para consulta presencial o mais brevemente possível, e em casos leves e moderados, para teleatendimento. Pacientes que não apresentavam descompensação psiquiátrica eram encaminhados à atenção básica ou tinham a opção de renovar a prescrição médica com o plantonista do próprio serviço.

Em virtude da ausência de recomendações específicas para teleatendimento psiquiátrico, alguns cuidados especiais foram tomados, como a exigência de contato de emergência, que deveria ser acionado imediatamente antes do início do atendimento remoto. Estabeleceu-se que os teleatendimentos fossem realizados pelos profissionais no espaço físico do serviço, em consultório, e registrados em prontuário. Nos casos em que durante o atendimento fossem observadas possíveis alterações do nível de consciência, risco de suicídio ou homicídio, sintomas psicóticos ou maníacos, o contato de emergência e o Samu eram acionados para encaminhamento imediato ao serviço de emergência (clínica ou psiquiátrica). Visando padronizar condutas e facilitar a tomada de decisão, algoritmos foram criados (Figuras 1 e 2). 
Figura 1. Algoritmo de decisão em situações de trauma e estresse

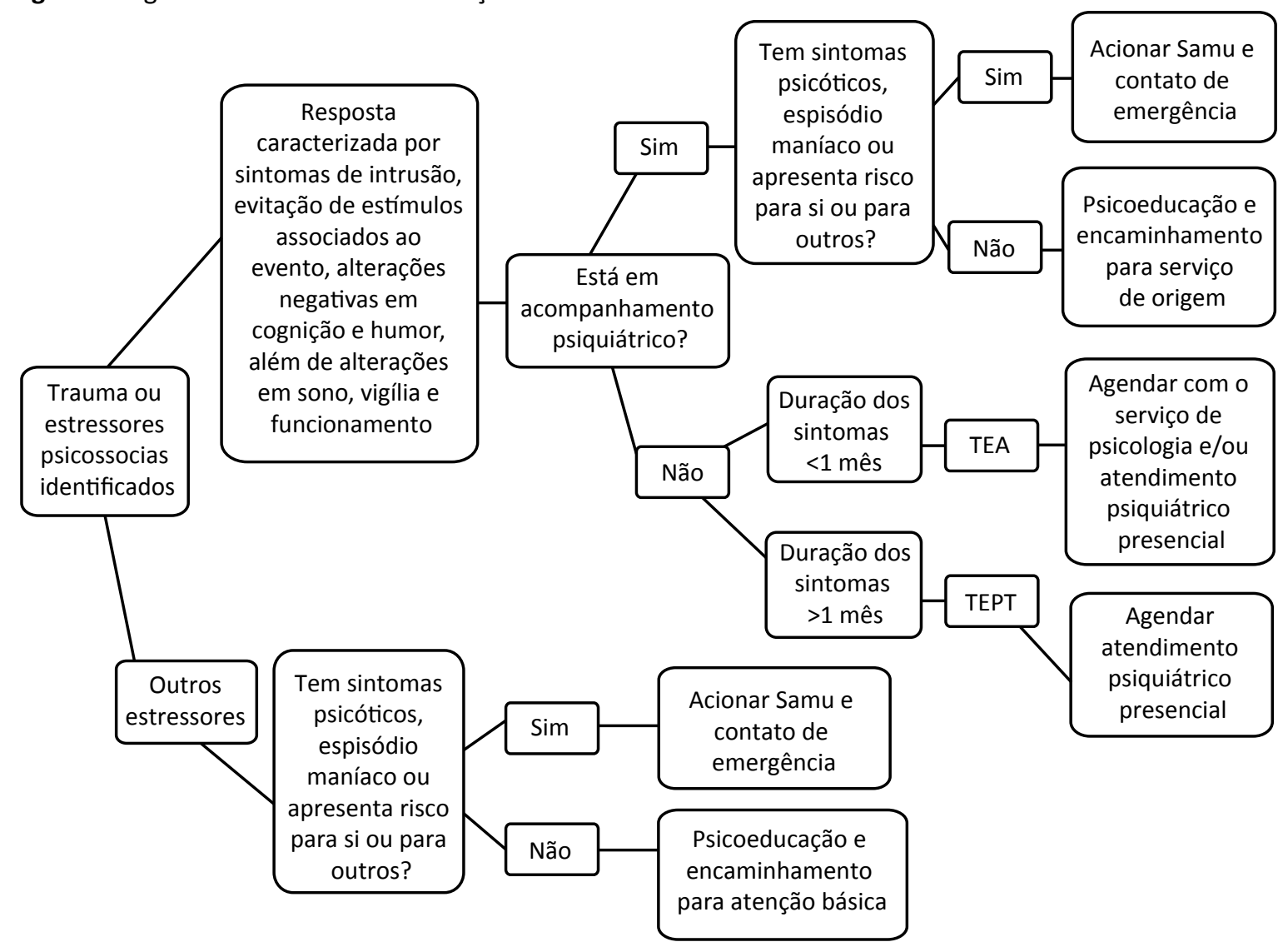

Samu: Serviço de Atendimento Móvel de Urgência; TEA: transtorno de estresse agudo; TEPT: transtorno de estresse pós-traumático

Figura 2. Algoritmo de decisão em sintomas do humor



Caps: Centro de Atenção Psicossocial; Caps AD: Centro de Atenção Psicossocial Álcool e Drogas; Samu: Serviço de Atendimento Móvel de Urgência 
A equipe não teve dificuldades para aderir ao teleatendimento, mas alguns usuários tiveram problemas para transmitir informações, especialmente os idosos e portadores de TMG, em decorrência de limitações cognitivas ou deficiências visuais e auditivas. Alguns indivíduos, apesar de não apresentarem restrições para o atendimento remoto, podem sentir desconforto ao transmitir informações por telefone ou videoconferência; portanto, não se sabe ainda se este é fator limitante para a avaliação sintomatológica. Neste ínterim, o relato familiar tem sido recurso indispensável para o monitoramento eficaz e a assertividade na tomada de decisão.

\section{Acolhimento em saúde mental em tempos de pandemia}

Experiências pregressas evidenciaram que durante períodos pandêmicos profissionais de saúde ficam mais suscetíveis a desenvolver transtorno de estresse agudo, TEPT e uso de substâncias. $\mathrm{Na}$ atual pandemia, tem-se verificado aumento na incidência de sintomas de transtornos mentais nesta população, sendo os depressivos os mais frequentes, seguidos dos sintomas de ansiedade, insônia e angústia. Os mais afetados entre os profissionais de saúde são mulheres, médicos e enfermeiros, principalmente os que atuam diretamente no combate à covid-19 25 . Com relação às medidas de prevenção do adoecimento mental, a terapia cognitivo-comportamental tem efeito positivo no sono, na fadiga, na depressão e no engajamento no trabalho. Outras medidas também têm se mostrado eficazes, como técnicas utilizando mindfulness, a intervenção breve focada no estresse e o treinamento de resiliência ${ }^{26}$.

O plano de ações para acolhimento dos trabalhadores, criado em caráter emergencial, ofereceu escuta qualificada para os profissionais do hospital universitário retratado neste estudo. Os interessados solicitavam atendimento via link disponibilizado nas mídias sociais oficiais, preenchiam formulário com dados sociodemográficos e de escala padronizada para avaliação de estresse e eram atendidos remotamente em até 48 horas, recebendo os primeiros cuidados psicológicos (PCP). A equipe multiprofissional realizava a triagem e direcionava o paciente a partir da avaliação dos eventos traumáticos e estressores associados, conforme algoritmo padronizado (Figura 3).

Figura 3. Algoritmo de triagem em teleatendimento

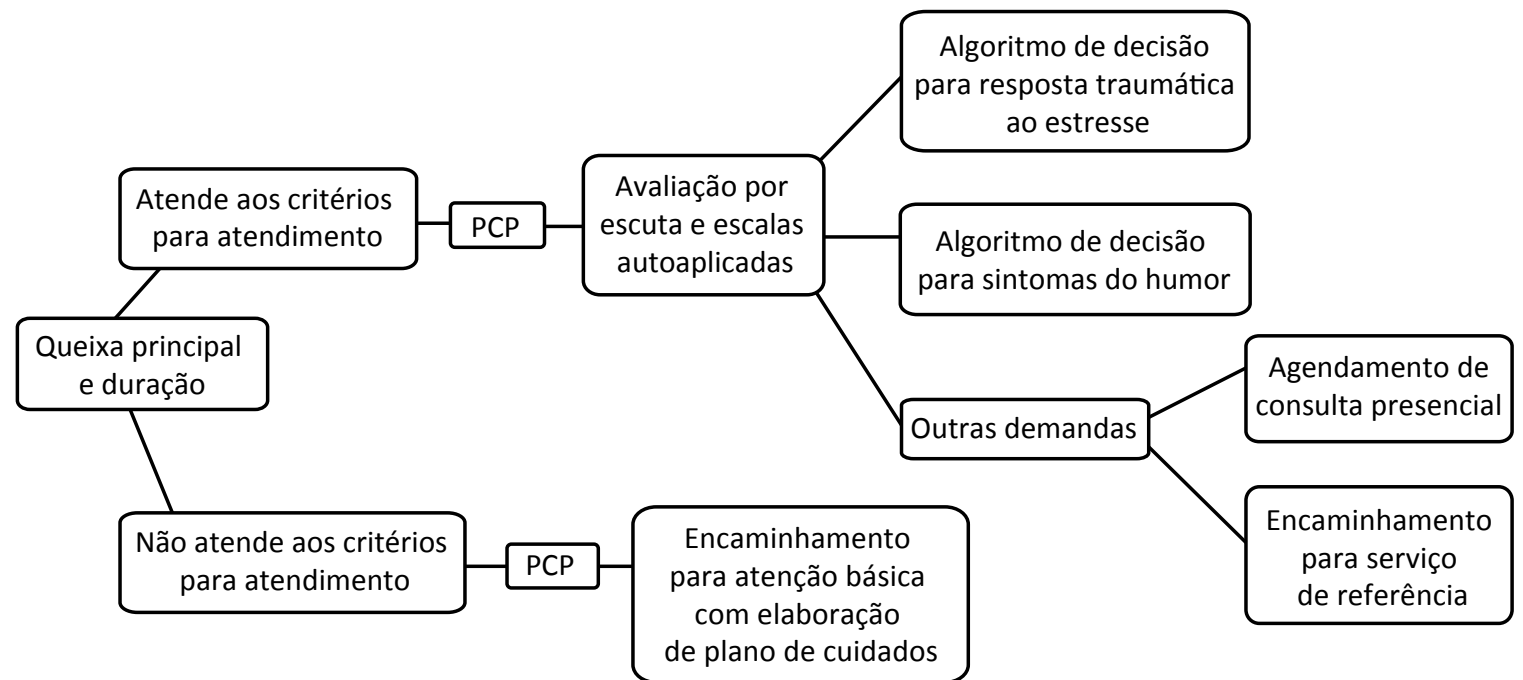

PCP: primeiros cuidados psicológicos

Os PCP têm como principal finalidade reduzir o estresse inicial causado pelos eventos potencialmente traumáticos e inserir o sujeito em estratégias de enfrentamento em curto e longo prazo ${ }^{27}$. É importante ressaltar que os PCP podem ser prestados por qualquer profissional, mas especialmente por aqueles formados e treinados em saúde mental. Tais intervenções devem responder às necessidades imediatas daqueles que querem compartilhar suas experiências e não devem ser consideradas tratamento psicoterápico, tampouco ter como objetivo o processamento emocional ${ }^{27}$. 
Durante a triagem, todos os profissionais eram acolhidos e recebiam os PCP. Ao serem identificados eventos traumáticos e estressores psicossociais (associados ou não a sintomas do humor), procedia-se à investigação ativa acerca de presença e duração de sintomas de intrusão, comportamentos evasivos relacionados ao evento, alterações cognitivas, sintomas do humor e alterações funcionais e do ciclo sono-vigília. Quando identificados sintomas do humor na ausência de evento traumático, o profissional era direcionado para atendimento de acordo com a gravidade. $O$ uso de substâncias psicoativas, risco de suicídio ou condições clínicas associadas eram ativamente investigados.

\section{Perspectivas para o futuro}

Ainda que amplamente utilizado durante a pandemia, o teleatendimento como recurso em saúde mental precisa ser validado, definindo-se também o perfil do paciente apto a recebê-lo de acordo com a gravidade da doença e seu acesso e habilidade no manuseio das tecnologias disponíveis. É ainda necessário validar ou criar escalas e instrumentos que melhorem a avaliação não presencial, e sobretudo treinar os profissionais para otimizar esse recurso.

Dadas as consequências econômicas da pandemia, estima-se que a taxa de desemprego aumentará em todo o mundo ${ }^{28,29}$, elevando, portanto, o número de suicídios e o surgimento ou agravamento de transtornos mentais ${ }^{29,30}$. Desta forma, é imprescindível que os serviços de saúde mental e as autoridades se mobilizem para formular medidas preventivas eficazes. Após a pandemia, e tendo superado os desafios trazidos por ela, certamente o atendimento terá sido aperfeiçoado, havendo profissionais mais conscientes de suas responsabilidades de entender, identificar e tratar transtornos. Do ponto de vista social, nos tornaremos mais capazes de formar redes de apoio e de entender o outro em suas reações, suas limitações e seu status quo.

\section{Referências}

1. Folha informativa covid-19: escritório da Opas e da OMS no Brasil. Organização Pan-Americana da Saúde [Internet]. 2020 [acesso 9 jun 2020]. Disponivel: https://bit.ly/2UAed9u

2. Conselho Federal de Medicina. Ofício CFM no 1.756/2020 - Cojur [Internet]. 19 mar 2020 [acesso 9 jun 2020]. Disponível: https://bit.ly/3kJJ3aE

3. Brasil. Ministério da Saúde. Portaria no 467, de 20 de março de 2020. Dispõe, em caráter excepcional e temporário, sobre as ações de telemedicina, com o objetivo de regulamentar e operacionalizar as medidas de enfrentamento da emergência de saúde pública de importância internacional previstas no art. 30 da Lei no 13.979, de 6 de fevereiro de 2020, decorrente da epidemia de covid-19. Diário Oficial da União [Internet]. Brasília, no 56-B, p. 1, 23 mar 2020 [acesso 9 jun 2020]. Seção 1 extra. Disponível: https://bit.ly/32QleaY

4. Agência Nacional de Vigilância Sanitária. Resolução da Diretoria Colegiada no 357, de 24 de março de 2020. Estende, temporariamente, as quantidades máximas de medicamentos sujeitos a controle especial permitidas em Notificações de Receita e Receitas de Controle Especial e permite, temporariamente, a entrega remota definida por programa público específico e a entrega em domicílio de medicamentos sujeitos a controle especial, em virtude da emergência de saúde pública de importância internacional (Espii) relacionada ao novo coronavírus (Sars-CoV-2). Diário Oficial da União [Internet]. Brasília, no 57-C, p. 2, 24 mar 2020 [acesso 9 jun 2020]. Seção 1 extra. Disponível: https://bit.ly/3kCzkCP

5. García-Lizana F, Muñoz-Mayorga I. Telemedicine for depression: a systematic review. Perspect Psychiatr Care [Internet]. 2010 [acesso 9 jun 2020];46(2):119-26. DOI: 10.1111/j.1744-6163.2010.00247.x

6. Rees CS, Maclaine E. A systematic review of videoconference-delivered psychological treatment for anxiety disorders. Aust Psychol [Internet]. 2015 [acesso 9 jun 2020];50(4):259-64. DOI: 10.1111/ ap.12122

7. Turgoose D, Ashwick R, Murphy D. Systematic review of lessons learned from delivering teletherapy to veterans with post-traumatic stress disorder. J Telemed Telecare [Internet]. 2018 [acesso 9 jun 2020];24(9):575-85. DOI: 10.1177/1357633X17730443

8. Liu S, Yang L, Zhang C, Xiang Y-T, Liu Z, Hu S, Zhang B. Online mental health services in China during the covid-19 outbreak. Lancet Psychiatry [Internet]. 2020 [acesso 9 jun 2020];7(4):e17-8. DOI: 10.1016/S2215-0366(20)30077-8

9. O'Brien M, McNicholas F. The use of telepsychiatry during covid-19 and beyond. Ir J Psychol Med [Internet]. 2020 [acesso 9 jun 2020]:1-6. DOI: 10.1017/ipm.2020.54

10. Shuja KH, Aqeel M, Jaffar A, Ahmed A. Covid-19 pandemic and impending global mental health implications. Psychiatr Danub [Internet]. 2020 [acesso 9 jun 2020];32(1):32-5. DOI: 10.24869/ psyd. 2020.32

11. Huremović $D$, editor. Psychiatry of pandemics: a mental health response to infection outbreak. Cham: Springer Nature; 2019.

12. Shinn AK, Viron M. Perspectives on the covid-19 pandemic and individuals with serious mental illness. $J$ Clin Psychiatry [Internet]. 2020 [acesso 9 jun 2020];81(3):20com13412. DOI: 10.4088/JCP.20com13412 
13. Dickerson F, Stallings CR, Origoni AE, Vaughan C, Khushalani S, Schroeder J, Yolken RH. Cigarette smoking among persons with schizophrenia or bipolar disorder in routine clinical settings, 1999-2011. Psychiatr Serv [Internet]. 2013 [acesso 9 jun 2020];64(1):44-50. DOI: 10.1176/appi.ps.201200143

14. Lan J, Ge J, Yu J, Shan S, Zhou H, Fan S et al. Structure of the Sars-CoV-2 spike receptor-binding domain bound to the ACE2 receptor. Nature [Internet]. 2020 [acesso 9 jun 2020];581:215-20. DOI: 10.1038/s41586-020-2180-5

15. Olfson M, Gerhard T, Huang C, Crystal S, Stroup TS. Premature mortality among adults with schizophrenia in the United States. Jama Psychiatry [Internet]. 2015 [acesso 9 jun 2020];72(12):1172-81. DOI: 10.1001/jamapsychiatry.2015.1737

16. Ho CSH, Chee CYI, Ho RCM. Mental health strategies to combat the psychological impact of covid-19 beyond paranoia and panic. Ann Acad Med Singap [Internet]. 2020 [acesso 9 jun 2020];49(3):155-60. Disponível: https://bit.ly/3a64n8n

17. Associação Brasileira de Psiquiatria. Ofício no 103/2020/ABP/SEC [Internet]. 31 mar 2020 [acesso 9 jun 2020]. Disponível: https://bit.ly/36Wcql6

18. ABP orienta: covid-19. Associação Brasileira de Psiquiatria [Internet]. 2020 [acesso 9 jun 2020]. Disponível: https://bit.ly/2IJcDzN

19. Holliday OJ. Para sistematizar experiências. 2a ed. rev. Brasília: Ministério do Meio Ambiente; 2006.

20. Leung JG, Nelson S, Barreto JN, Schiavo DN. Necrotizing pneumonia in the setting of elevated clozapine levels. J Clin Psychopharmacol [Internet]. 2016 [acesso 9 jun 2020];36(2):176-8. DOI: 10.1097/JCP.0000000000000470

21. Pereira MD, Oliveira LC, Costa CFT, Bezerra CMO, Pereira MD, Santos CKA, Dantas EHM. A pandemia de covid-19, o isolamento social, consequências na saúde mental e estratégias de enfrentamento: uma revisão integrativa. Res Soc Dev [Internet]. 2020 [acesso 9 jun 2020];9(7):e652974548. DOI: $10.33448 /$ rsd-v9i7.4548

22. Hagerty SL, Williams LM. The impact of covid-19 on mental health: the interactive roles of brain biotypes and human connection. Brain Behav Immun Health [Internet]. 2020 [acesso 9 jun 2020];5:100078. DOI: 10.1016/j.bbih.2020.100078

23. Luykx JJ, Van Veen SMP, Risselada A, Naarding P, Tijdink JK, Vinkers $\mathrm{CH}$. Safe and informed prescribing of psychotropic medication during the covid-19 pandemic. Br J Psychiatry [Internet]. 2020 [acesso 9 jun 2020];217(3):471-4. DOI: 10.1192/bjp.2020.92

24. Volmer AL, Bridi Filho CAN. Protocolo de Manchester em pauta: como este aborda as questões de saúde mental? RDS Ciênc Hum [Internet]. 2016 [acesso 9 jun 2020];17(1):103-16. DOI: 10.37780/ch.v17i1.2022

25. Lai J, Ma S, Wang Y, Cai Z, Hu J, Wei $\mathrm{N}$ et al. Factors associated with mental health outcomes among health care workers exposed to coronavirus disease 2019. Jama Netw Open [Internet]. 2020 [acesso 9 jun 2020];3(3):e203976. DOI: 10.1001/jamanetworkopen.2020.3976

26. Melnyk BM, Kelly SA, Stephens J, Dhakal K, McGovern C, Tucker S et al. Interventions to improve mental health, well-being, physical health, and lifestyle behaviors in physicians and nurses: a systematic review. Am J Health Promot [Internet]. 2020 [acesso 5 dez 2020];34(8):929-41. DOI: $10.1177 / 0890117120920451$

27. International Federation of Red Cross and Red Crescent Societies. Psychosocial Centre. Primeiros socorros psicológicos remotos durante o surto de covid-19 [Internet]. Copenhagen: IFRC; 2020 [acesso 5 out de 2020]. Disponível: https://bit.ly/3pw2W8J

28. Almost 25 million jobs could be lost worldwide as a result of covid-19, says ILO. International Labour Organization [Internet]. 18 mar 2020 [acesso 9 jun 2020]. Disponível: https://bit.ly/2INCgz2

29. Kawohl W, Nordt C. Covid-19, unemployment, and suicide. Lancet Psychiatry [Internet]. 2020 [acesso 9 jun 2020];7(5):389-90. DOI: 10.1016/S2215-0366(20)30141-3

30. Klomek AB. Suicide prevention during the covid-19 outbreak. Lancet Psychiatry [Internet]. 2020 [acesso 9 jun 2020];7(5):390. DOI: 10.1016/S2215-0366(20)30142-5

\title{
Participação dos autores
}

Todos os autores discutiram ativamente o tema, elaboraram e revisaram o manuscrito.

\section{Correspondência}

Alfredo José Minervino - Rua Giácomo Porto, 145, Edifício Torre Imperial, apt. 1.601, Miramar CEP 58032-110. João Pessoa/PB, Brasil.

\author{
Alfredo José Minervino - Mestre - alfredominervino@hotmail.com \\ (iD) $0000-0002-6454-4598$ \\ Marina Barbosa de Oliveira - Graduada - marinadeoliveira@hotmail.com \\ (iD) 0000-0002-5253-4376 \\ Kaio Aranda Lima da Cunha - Graduado - kaio_alc@hotmail.com \\ (iD) 0000-0001-8263-7862 \\ Ygor Thalles Almeida Bereza - Graduado - ygor.bereza@gmail.com \\ (iD) $0000-0002-5940-2472$
}

